\title{
Low Contrast Gray Image Enhancement using Particle Swarm Optimization (PSO) with DWT
}

\author{
Aarti Pareyani \\ Department of Electronics and Communication \\ Engineering \\ Jabalpur Engineering College, Jabalpur (M.P.), \\ India
}

\author{
Agya Mishra \\ Department of Electronics and Communication \\ Engineering \\ Jabalpur Engineering College, Jabalpur (M.P.), \\ India
}

\begin{abstract}
Low contrast image enhancement is the task of applying certain transformations to an input image such as to obtain a visually more recovered, more detailed, or less noisy output image. In this paper image enhancement is considered as an optimization problem and the algorithm Particle Swarm Optimization (PSO) along with DWT is used to solve it.The objective of the proposed algorithm is to maximize an objective fitness criterion in order to enhance the contrast and detail in an image by adapting the parameters of a novel extension to a local enhancement technique. The Entropy Gain and objective criterion has been used as a comparison parameter for proposed image enhancement method.
\end{abstract}

\section{Keywords}

Particle swarm optimization, discrete wavelet transform, Low contrast enhancement, fitness function, entropy gain

\section{INTRODUCTION}

One of the most common defects of photographic or digital images is poor contrast resulting from reduced, and nonlinear, image amplitude range [11] .Producing digital images with good contrast and detail is a strong requirement in several areas. The principle objective of enhancement is to process an image so that the result is more suitable than the original image.Image enhancement approaches fall into two broad categories: spatial domain methods and frequency domain methods. The term spatial domain refers to the image plane itself, and approaches in this category are based on direct manipulation of pixels in an image. Frequency domain processing techniques are based on modifying the Fourier transform of an image.Visual evaluation of image quality is a highly subjective process, thus making the definition of a "good image" an elusive standard by which to compare algorithm performance. When the problem is one of processing images for machine perception, the evaluation task is somewhat easier [12].

Particle Swarm Optimization (PSO) is one of the modern heuristic algorithms that can be applied to nonlinearand noncontinuous optimization problems. It is a population-based stochastic optimization technique for continuous nonlinear functions.PSO term refers to a relatively new family of algorithms that may be used to find optimal (or near optimal) solutionsto numerical and qualitative problems [4].PSO was discovered through simulation of a simplified bird flocking model. Dr. Kennedy and Dr. Eberhart stated in [5]"'Particle swarm optimization has roots in two main component methodologies. Perhaps more obvious are its ties to artificiallife (A-life) in general, and to bird flocking, fishschooling, and swarming theory in particular.Particle swarm optimization asdeveloped by [Kennedy and Eberhart] comprises a very simple concept, and paradigms can be implemented in a few lines of computer code. It requires only primitive mathematical operators, and is computationally inexpensivein terms of both memory requirements and speed.Early testing has found the implementation to be effective with several kinds of problems.

A discrete wavelet transform (DWT) is a wavelet transform in which the wavelets are discretely sampled for numerical analysis and functional analysis. The advantage of DWT is, it captures both frequency and time information. Discrete wavelet transform (DWT) decompose signals into sub-bands with smaller bandwidths and slower sample rates namely Low-Low (LL), Low-High (LH), High-Low (HL), andHighHigh $(\mathrm{HH})$. With this four sub-bands are obtained from one level of transform - first low-pass sub-band having the coarse approximation of the source image called LL sub-band, and three high pass sub-bands that exploitimage details across different directions - HL for horizontal, LH for vertical and $\mathrm{HH}$ for diagonal details.

In this work a new contrast enhancement algorithm is proposed using PSO and DWT. The rest of the paper is organized as follows: In Section II, theory of PSO and DWT (transformation and evaluation function) are described. In Section III, proposed algorithm is discussed. In Section IV, in experimental results, the effectiveness of the proposed scheme is checked by using Entropy gain and fitness value and finally in Section V, conclusion of the work is made.

\section{THEORY}

\subsection{PSO Algorithm}

Particle Swarm Optimization was introduced by Dr. Russell C. Eberhart and Dr. James Kennedy in 1995.This optimization algorithm is a multi agent based search strategy modelled on the social behaviour of organisms such as bird flocking and fish schooling.PSO as an optimization tool provides a population based search procedure in which individuals called particles change their position with time [1].In PSO, each single solution is a "particle". All of the particles have fitness values which are evaluated by the objective function to be optimized, and have velocities which direct the flying of the particles. The particles fly through the problem space by following the personal and global best particles.

The swarm is initialized with a group of random particles and it then searches for optima by updating through iterations. In every iteration, each particle is updated by following two "best" values. The first one is the best solution of each particle achieved so far. This value is known as pbest solution. Another one is that, best solution tracked by any particle among all generations of the swarm. This best value is known as gbest solution. These two best values are responsible to drive the particles to move to new better position. 
Particle Swarm optimization technique has mainly two primary operators:

\section{- Velocity update}

- Position update: During each generation each particle is accelerated toward the particle's previous best position (pbest) and the global best (gbest) position and new velocity value for each particle is calculated.

After finding the two best values, a particle updates its velocity and position with the help of the following equations:

$$
\begin{gathered}
v_{i}^{t+1}=w * v_{i}^{t}+c_{1} * r_{1} *\left[\text { pbest }_{i}^{t}-X_{i}^{t}\right]+ \\
c_{2} * r_{2} *\left[\text { gbest }-X_{i}^{t}\right] \\
X_{i}^{t+1}=X_{i}^{t}+v_{i}^{t+1}
\end{gathered}
$$

Where $X_{i}^{t}$ and $v_{i}^{t}$ denotes the position and velocity of $i^{t h}$ particle at time instance $t, w$ is inertia weight at $t^{\text {th }}$ instant oftime. Maximum and minimum value for this inertia weight is set to two and zero respectively, which is same for all particles. Inertia value $w$ is calculated as follows:

$$
w=w_{\max }-\frac{\left(w_{\max }-w_{\min }\right)}{t_{\max }} \times t
$$

Where, $t$ is the $i^{t h}$ iteration and $t_{\max }$ is the total number of iteration. Parameters $c_{1}$ and $c_{2}$ are positive acceleration constants given a random number in $[0,2]$. These parameters are fixed for each particle throughout its life. $r_{1}$ and $r_{2}$ are random values generated in the range $[0,1]$. pbest ${ }_{i}$ is the best solution of $i^{t h}$ individual particle over its flight path, gbest is the best particle obtained over all generations so far.

\subsection{Discrete Wavelet Transform (DWT)}

The discrete wavelet transform (DWT) is an implementation of the wavelet transform using a discrete set of the wavelet scales for numerical analysis and functional analysis. The decomposition of images into various frequency ranges permits the isolation of the frequency into certain sub-bands. This process results in isolating small changes in an image mainly in low frequency sub-band images. The 2D wavelet decomposition of an image is performed by applying 1D DWT along the rows of the image first, and, then, the results are decomposed along the columns. This Decomposition results in four decomposed sub-band images referred to as low-low (LL), low-high (LH), high-low (HL), and high-high (HH).

\subsection{Transformation Function}

For image enhancement task, a transformation function is required which will take the intensity value of each pixel from the input image and generate a new intensity value for the corresponding pixel to produce the enhanced image. To evaluate the quality of the enhanced image automatically, an evaluation function is needed which will tell about the quality of the enhanced image. Image enhancement done on spatial domain uses a transform function which generates a new intensity value for each pixel of the $\mathrm{M} * \mathrm{~N}$ original image to generate the enhanced image, where $\mathrm{M}$ denotes the number of columns and $\mathrm{N}$ denotes the number of rows. The enhancement process can be denoted by:

$$
g(x, y)=T(f(x, y))
$$

where $f(x, y)$ is the gray value of the $(x, y)^{\text {th }}$ pixel of the input image and $g(x, y)$ is the gray value of the $(x, y)^{\text {th }}$ pixel of the enhanced image. $\mathrm{T}$ is the transformation function.Local enhancement methods apply transformation on a pixel considering intensity distribution among its neighbouring pixels [10]. The function used here is designed in such a way that takes both global as well as local information to produce the enhanced image. Local information is extracted from a user defined window of size $n \times n$. The transformation is defined as:

$$
\begin{gathered}
g(x, y)=\left[K \frac{D}{\sigma(x, y)+b}\right][f(x, y)-c * m(x, y)]+ \\
m(x, y)^{a}
\end{gathered}
$$

In (5), $\mathrm{m}(\mathrm{x}, \mathrm{y})$ and $\sigma(\mathrm{x}, \mathrm{y})$ are the gray-level mean and standard deviation computed for the pixels inside a neighbourhood window centred at $(\mathrm{x}, \mathrm{y})$ and having $\mathrm{n} \times \mathrm{n}$ pixels. $\mathrm{D}$ is the global mean of the input image.With the transformation stated in eq. (5) contrast of the image can be stretched considering local mean as the centre of stretch. The last term $\mathrm{m}(\mathrm{x}, \mathrm{y})$ a has brightening \& smoothing effect thus smooths the output image and the four parameters introduced in the transformation function i.e. $a, b, c \& K$ are the parameters of the enhancement function and the small variation in their value produces a large variation in the processed output image and thus the value of these parameters are precisely set. The approximate range of these parameters is defined as: $\mathrm{a}[0,1.5]$; $\mathrm{b}[0,(\mathrm{D} / 2)] ; \mathrm{c}[0,1] ; \mathrm{K}[.5,1.5]$.

\subsection{Evaluation Criterion}

In order to evaluate the performance of the proposed algorithm and the quality of an enhanced image an objective function is required which tells about the quality of the output image.It is observed that compared to the original image good contrast enhanced image has more number of edgels i.e. number of edge pixels and enhanced version should have a higher intensity of the edges [1]. But these two measures are not sufficient to test an enhanced image and that why one more performance measure is taken into account i.e. entropy value of the image. Entropy value reveals the information content in the image. If the distribution of the intensities is uniform, then the histogram is equalized and the entropy of the image will be more. Thus, the objective function considered uses three performance measures i.e. entropy value, sum of edge intensities value and number of edgels (edge pixels) and is defined as:-

$$
F\left(I_{e}\right)=\log \left(\log \left(E\left(I_{e}\right)\right) * \frac{n_{\text {edges }\left(I_{e}\right)}}{M * N} * H\left(I_{e}\right)\right.
$$

In the above mentioned equation Ie is the enhanced image resulted from the transformation function defined above. In eq. (6) the edges or edgels can be detected by many efficient edge detector algorithms such as Sobel, Laplacian, Canny etc. In this study Canny is used as an automatic threshold detector. Canny's edge detection algorithm is computationally more expensive compared to Sobel, Prewitt and Robert's operator. However, the Canny's edge detection algorithm performs better than all these operators under almost all scenarios and thus in this work Canny edge operator is used. $\mathrm{E}(\mathrm{Ie})$ is the sum of $\mathrm{Mx}$ N pixels intensities of canny edge images. $\mathrm{n}_{\text {edges }}$ is the number of pixels, whose intensity value is greater than a threshold in canny edge image.Entropy of the enhanced image Ie is given by:-

$$
H\left(I_{e}\right)=-\sum_{i=0}^{255} e_{i}
$$

Where $e_{i}=h_{i} \log _{2}\left(h_{i}\right)$ if $h_{i} \neq 0$ otherwise $e_{i}=0$. And $h_{i}$ is the probability of occurrence of intensity value of image. 


\section{PROPOSED ALGORITHM PSO-DWT}

In the proposed method an enhanced image produced from a transformation function which incorporates both global and local information of the input image defined in eq. (5) is used. The function also contains four parameters namely a, b, c, k which are used to produce diverse result and help to find the optimal one according to the objective function. These four parameters have their defined range described above. Now the aim is to find the best set of values for these four parameters which can produce the optimal result and to perform this work PSO is used. P number of particles are initialized, each with four parameter $\mathrm{a}, \mathrm{b}, \mathrm{c}$ and $\mathrm{k}$ by the random values within their range and corresponding random velocities. It means position vector of each particle $\mathrm{X}$ has four component $\mathrm{a}, \mathrm{b}$, c and $\mathrm{k}$. Now using these parameter values, each particle generates an enhanced image. Quality of the enhanced image is calculated by an objective function defined in eq. (6) which is termed as fitness of the particle. Fitness value of all the enhanced images generated by all the particles is calculated. From these fitness values pbest and gbest are found. With the help of these best values, component wise new velocity of each particle is calculated to get the new solution. In this way new positions of particles are created for generations. When the process is completed the enhanced image is created by the global best (gbest) particle. To remove the noise present in the image obtained at the output of the PSO algorithm, it is decomposed into four different frequencies sub bands namely, LL (lowlow), LH (low-high), HL (high-low) and HH (high-high) using DWT. The processed image obtained at the end is not only enhanced but also has sharp edges. The pseudo code for the proposed algorithm is shown in Fig. 1 below.

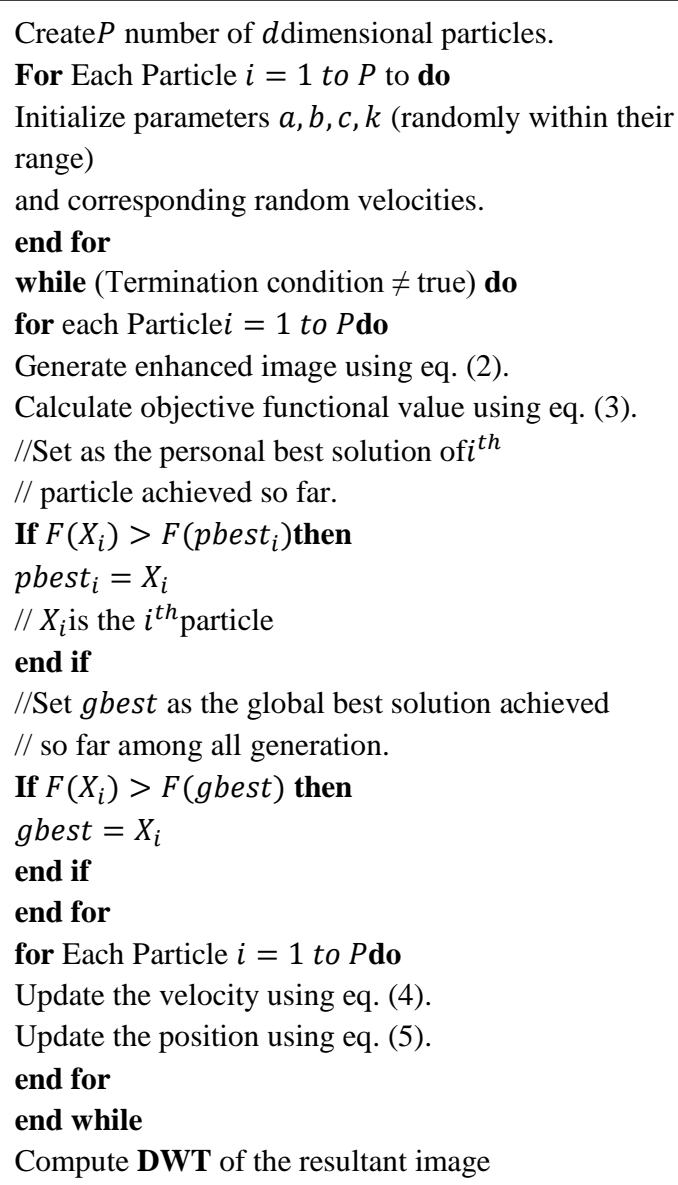

\subsection{Entropy Gain}

The performance of the algorithm is evaluated using the fitness function given in eq. (3) and the entropy gain. The entropy gain is calculated using the function as defined below:

$$
\text { Entropy_gain }=H\left(I_{e}\right)-H(I)
$$

Where $H\left(I_{e}\right)$ and $H(I)$ are the entropy of the enhanced image and the input image respectively.

\section{EXPERIMENTAL RESULTS}

In this section, the proposed algorithm is evaluated by couple of case studies. Two images are considered in case studies: one of size $259 \times 194$ of Jabalpur Engineering College, premises and other is the inbuilt cameraman image of size $256 \times 256$.The parameters a,b,c \& $\mathrm{K}$ in eq.(5) are initialized within the range $[0,1.5] ;[0,(\mathrm{D} / 2)] ;[0,1]$ and $[0.5,1.5]$ respectively.

\subsection{Case study 1:}

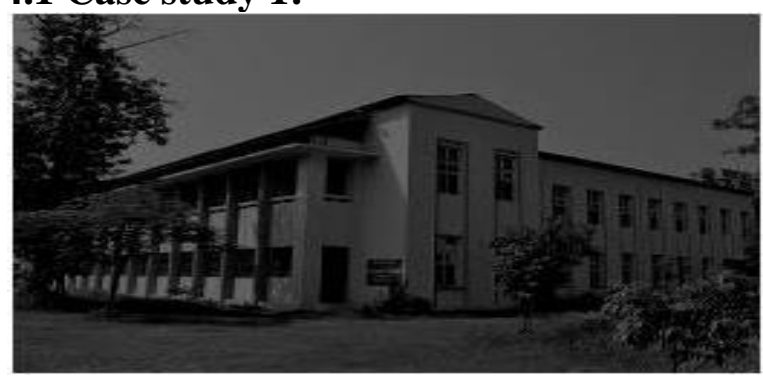

(a) Low contrast Image

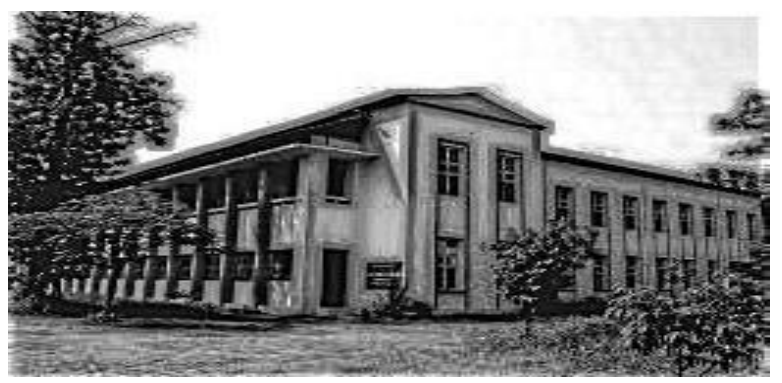

(b) Enhanced Image

Fig. 2 Images of case study 1

\subsubsection{Parameter Based Analysis:}

In this case study, input image taken is shown in fig 2(a), which when processed results in an image as shown in fig 2(b). Fig 3 shows the effect of $P$ (particle size) on processed image and their respective entropy gain and fitness value.

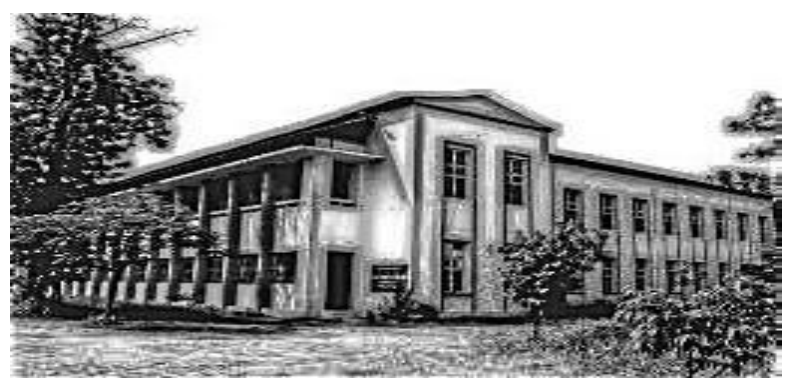

$\mathrm{P}=25$; Fitness=2.9427; Entropy_Gain=0.6829

Fig. 1 Pseudo Codes 


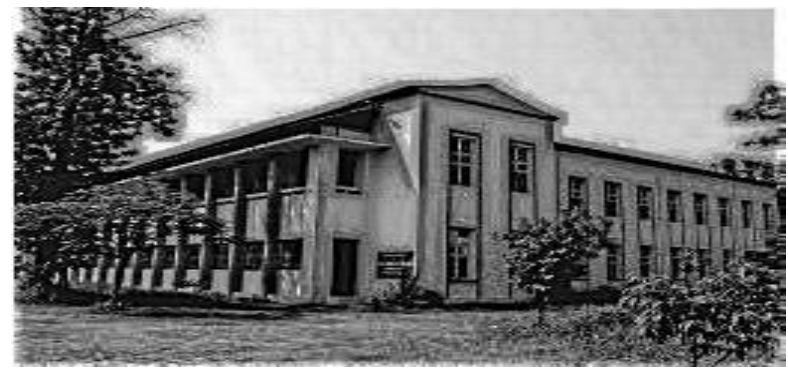

P=30; Fitness=2.9961; Entropy_Gain=0.8147

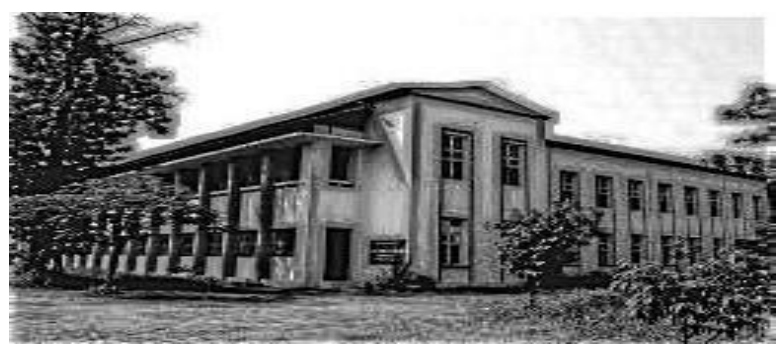

$\mathrm{P}=35 ;$ Fitness=2.9969; Entropy_Gain $=0.8522$

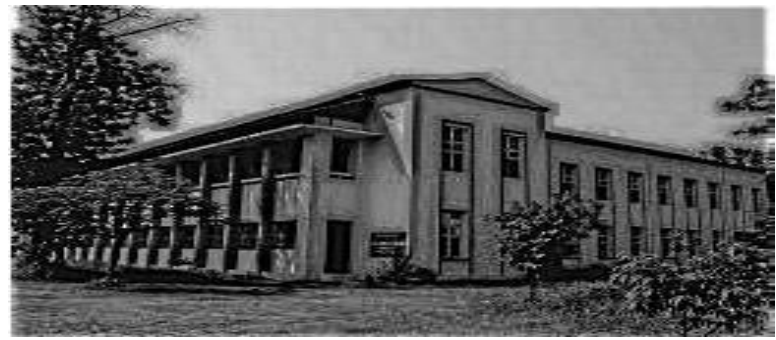

$\mathrm{P}=40 ;$ Fitness=2.9901; Entropy_Gain=0.8469

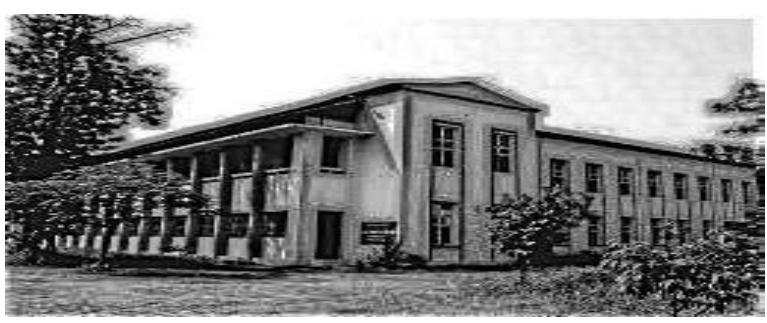

$\mathrm{P}=45$; Fitness=2.9902; Entropy_Gain=0.7928

Fig.3 Effect of Particle size on Processed Image

\subsubsection{Variation of Entropy Gain and Fitness} Function with Particle Size

Figure 4(a) shows the plot between particle sizeand entropy gain and fig. 4(b) shows the plot between particle size and fitness parameter. The highest Fitness value and Entropy gain is obtained for particle size 35 which are 2.9969 and 0.8522 , implies that it is the best result of case study 1 .

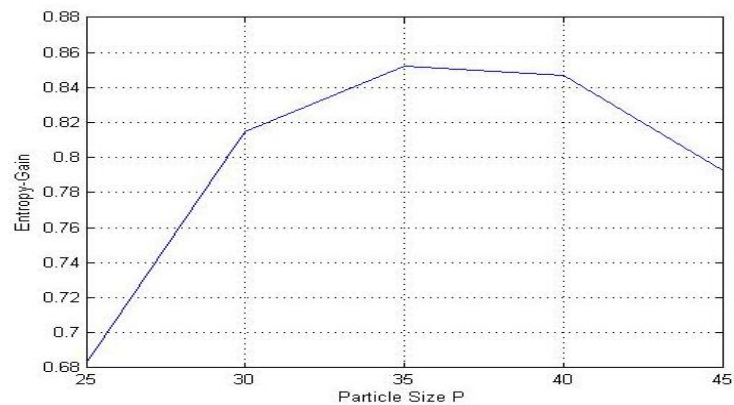

(a) Particle Size Vs Entropy_Gain

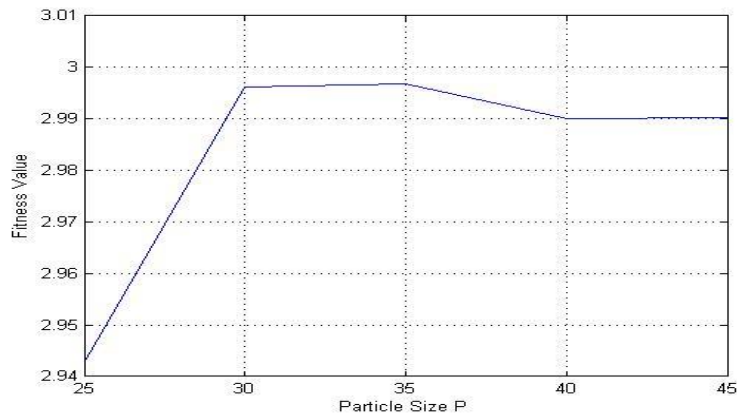

(b) Particle Size Vs Fitness Value

Fig.4 Plots of case study 1

\subsection{Case Study 2:}

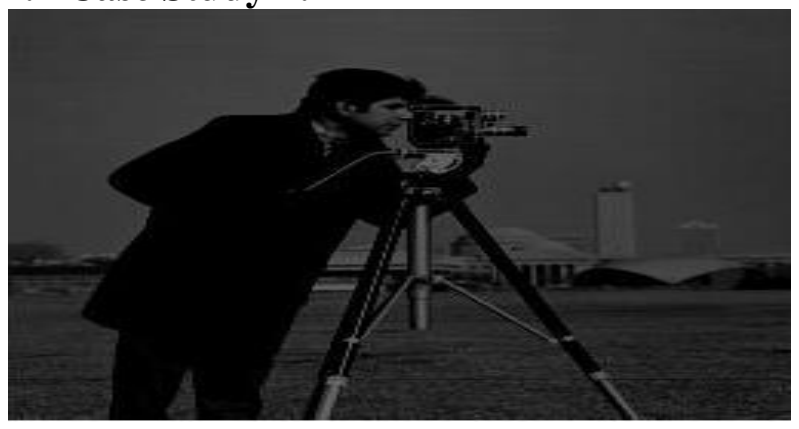

(a) Low Contrast Image

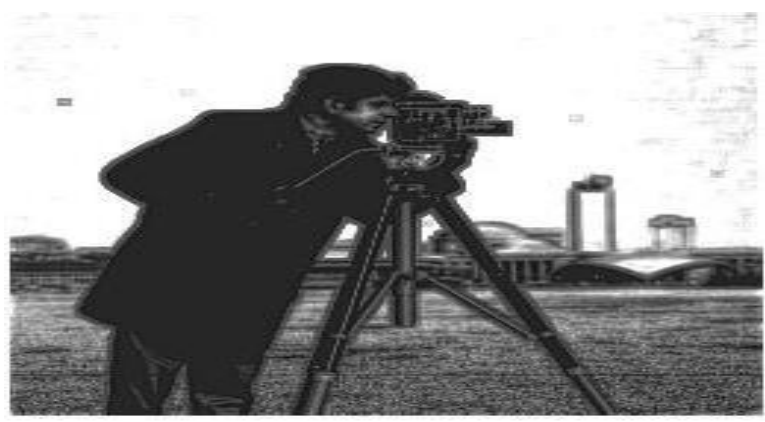

(b) Enhanced Image

Fig. 5 Images of case study 2

\subsubsection{Parameter Based Analysis:}

In this case study, input image taken is shown in fig 5(a), which when processed results in an image as shown in fig 5(b). Fig. 6 shows the effect of $P$ (particle size) on processed image and their respective entropy gain and fitness value.

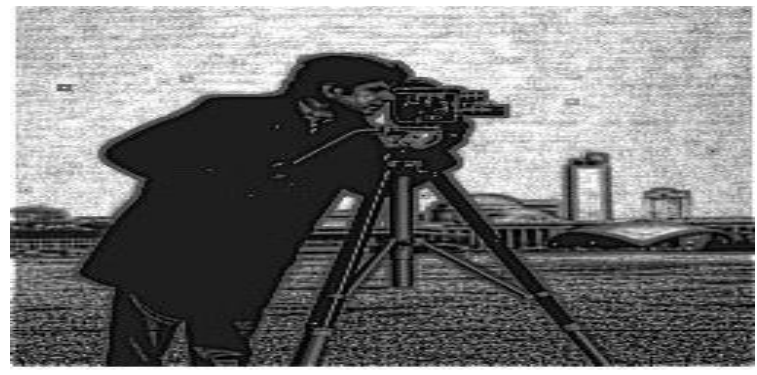

$\mathrm{P}=15$; Fitness=2.5546; Entropy_Gain=0.0.6981 


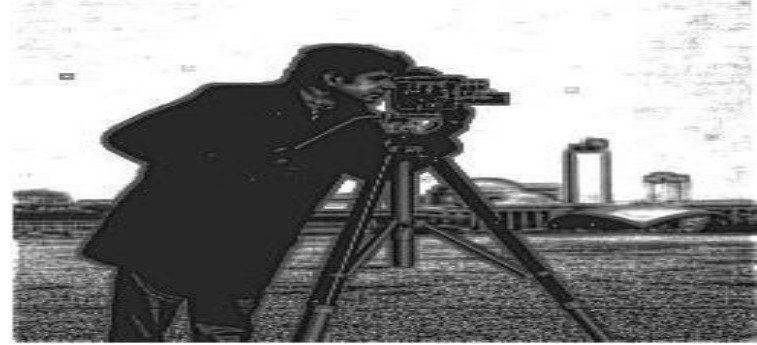

$\mathrm{P}=20$; Fitness=2.4822; Entropy_Gain $=0.8669$

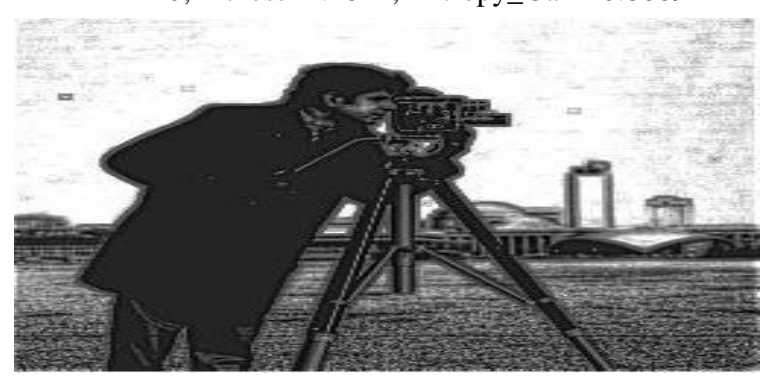

$\mathrm{P}=25$; Fitness=2.5888; Entropy_Gain=0.1.0750

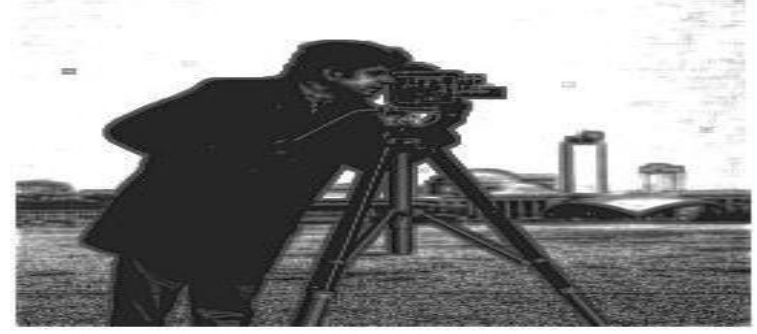

$\mathrm{P}=30$; Fitness=2.6234; Entropy_Gain=1.2915

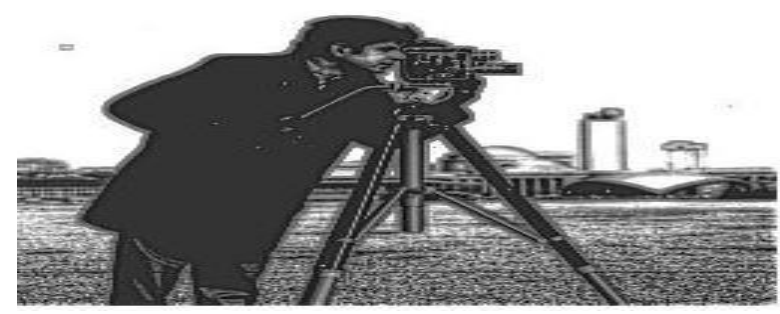

$\mathrm{P}=35$; Fitness=2.5401; Entropy_Gain=0.9029

Fig.6 Effect of Particle size on Processed Image

\subsubsection{Variation of Entropy Gain and Fitness} Function with Particle Size

Figure 7(a) shows the plot between particle size and entropy gain and fig 7(b) shows the plot between particle size and fitness parameter. The highest Fitness value and Entropy gain is obtained for particle size 30 which are 2.6234 and 1.2915, implies that it is the best result of case study 2 .

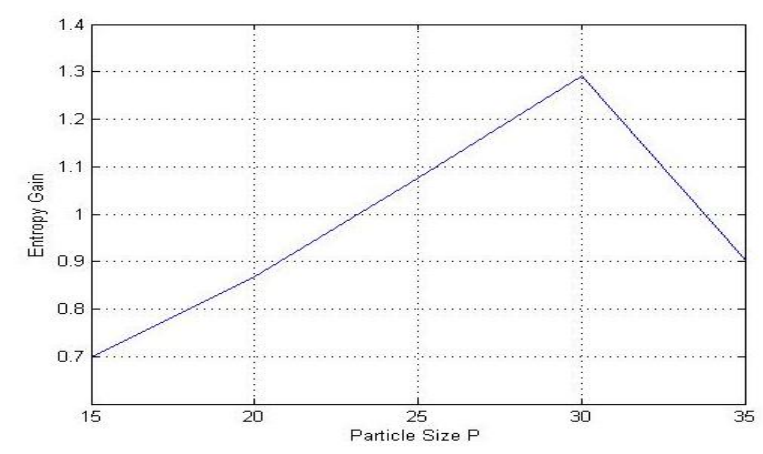

(a) Particle Size Vs Entropy_Gain

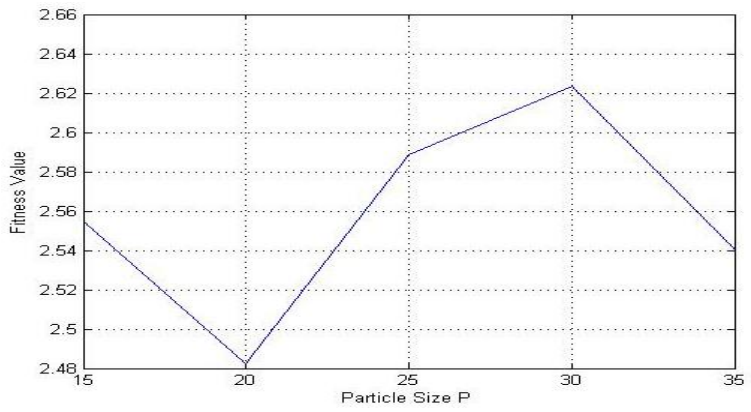

(b) Particle Size Vs Fitness Value

Fig.7 Plots of case study 2

\subsection{Comparison with Existing Algorithm}

In this section, the proposed PSO with DWT algorithm is compared with the existing PSO algorithm. Table 1 compares the Fitness value obtained from PSO algorithm [2] and the proposed algorithm for the two images.Table 1 shows that the result obtained from the proposed algorithm is better as compared to the existing algorithm.

Table1 Fitness value comparison with PSO algorithm [2]

\begin{tabular}{|c|c|c|c|}
\hline & Parameter & $\begin{array}{c}\text { PSO } \\
\text { Algorithm }\end{array}$ & $\begin{array}{c}\text { Proposed } \\
\text { Algorithm }\end{array}$ \\
\hline $\begin{array}{c}\text { Case } \\
\text { Study 1 }\end{array}$ & Fitness & 2.38565 & 2.9969 \\
\hline $\begin{array}{c}\text { Case } \\
\text { Study 2 }\end{array}$ & Fitness & 1.9363 & 2.6234 \\
\hline
\end{tabular}

Table 2 compares the Entropy Gain obtained from the PSO algorithm [3] and the proposed algorithm. The table concludes that the proposed algorithm have higher entropy gain than the existing algorithm.

Table 2 Entropy gain comparison with PSO algorithm [3]

\begin{tabular}{|c|c|c|c|}
\hline & Parameter & $\begin{array}{c}\text { PSO } \\
\text { Algorithm }\end{array}$ & $\begin{array}{c}\text { Proposed } \\
\text { Algorithm }\end{array}$ \\
\hline $\begin{array}{c}\text { Case } \\
\text { Study 1 }\end{array}$ & Entropy_gain & 0.435 & 0.8522 \\
\hline $\begin{array}{c}\text { Case } \\
\text { Study 2 }\end{array}$ & Entropy_gain & 0.722 & 1.2915 \\
\hline
\end{tabular}

\section{CONCLUSIONS}

The Proposed algorithm has been successfully implemented. The presented work is found efficient in enhancing the contrast of an image. In this paper, a PSO with DWT algorithm is proposed and implemented for low contrast image enhancement. Experimental results and comparison with the existing algorithms concludes that the proposed algorithm performs better and is quite effective for contrast enhancement. The values of fitness function and entropy gain obtained can further be improved by using different wavelets and by increasing the level of DWT decomposition in future.

\section{REFERENCES}

[1] Apurba Gorai and Ashish Ghosh, 2009 Gray-level Image Enhancement By Particle Swarm Optimization, IEEE World Congress on Natural \& Biologically Inspired Computing (NaBIC).

[2] Nirmal Singh, Maninder Kaur and K.V.P Singh,2013 Parameter Optimization in Image Enhancement Using PSO, American Journal of Engineering Research (AJER), Vol-2, pp. 84-90. 
[3] Ngaiming Kwok,Haiyan Shi,Gu Fang and Quang Ha, 2013 Adaptive Scale Adjustment Design of Unsharp Masking Filters for Image Contrast Enhancement, IEEE Proceeding of the International Conference on Machine Learning and Cybernetics, Tianjin.

[4] Malik Braik, Alaa Sheta and Aladdin Ayesh, 2007 Image Enhancement Using Particle Swarm Optimization, Proceedings of World Congress on Engineering Vol. 1, pp. 2-4,London UK.

[5] J. Kennedy and R. C. Eberhart, 1995 Particle swarm optimization, Proceedings of IEEE International Conference on Neural Networks (Perth, Australia), IEEE Service Centre, Piscataway, NJ, vol. 5, no. 3, pp. 19421948.

[6] C. Munteanu and A. Rosa, 2001 Towards Automatic Image Enhancement Using Genetic Algorithms, LaSEEB-ISR-Instituto Superior Tcnico.
[7] F. Saitoh, 1999 Image Contrast Enhancement Using Genetic Algorithm, IEEE International Conference on Volume: 4, pp. 899 - 904.

[8] C. Munteanu, A. Rosa, 2004 Gray-scale enhancement as an automatic process driven by evolution", IEEE Transaction on Systems, Man and Cybernatics-Part B: Cybernetics, vol. 34, no. 2, pp. 1292-1298.

[9] William K. Pratt, 2001 Digital Image Processing, John Wiley and Sons, Inc.

[10] Cristain Ordyo Casado. Assoc. Prof. Antoaneta Popova, 2010 Image Enhancement Methods, Sofia.

[11] Anish Kumar Vishwakarma, Agya Mishra, K. Gaurav and A. Katariya, 2012 Illumination Reduction For Low Contrast Color Image Enhancement With Homorphic Filtering Technique, IEEE International Conference on Communications Systems and Network Technologies (CSTN), pp. 171-173. 